\section{(2) OPEN ACCESS}

\title{
Defining indicators for drug overdose emergency department visits and hospitalisations in ICD-10-CM coded discharge data
}

\author{
Alana Vivolo-Kantor () , Emilia Pasalic, Stephen Liu, Pedro D Martinez, \\ Robert Matthew Gladden, Overdose Morbidity Team
}

\begin{abstract}
- Additional material is published online only. To view, please visit the journal online (http://dx.doi.org/10.1136/ injuryprev-2019-043521).
\end{abstract}

National Center for Injury Prevention and Control, Centers for Disease Control and Prevention, Atlanta, Georgia, USA

\section{Correspondence to} Dr Alana Vivolo-Kantor, National Center for Injury Prevention and Control, Centers for Disease Control and Prevention, Atlanta, GA 30341, USA; goz4@cdc.gov

Received 16 December 2019 Revised 17 September 2020 Accepted 19 November 2020

\section{Check for updates}

(C) Author(s) (or their employer(s)) 2021. Re-use permitted under CC BY-NC. No commercial re-use. See rights and permissions. Published by BMJ.

To cite: Vivolo-Kantor A Pasalic E, Liu S, et al. Inj Prev 2021;27:i56-i61.

\begin{abstract}
Introduction The drug overdose epidemic has worsened over the past decade; however, efforts have been made to better understand and track nonfatal overdoses using various data sources including emergency department and hospital admission data from billing and discharge files.
\end{abstract}

Methods and findings The Centers for Disease Control and Prevention (CDC) has developed surveillance case definition guidance using standardised discharge diagnosis codes for public health practitioners and epidemiologists using lessons learnt from CDC's funded recipients and the Council for State and Territorial Epidemiologists (CSTE) International Classification of Diseases, 10th Revision, Clinical Modification (ICD-10CM) Drug Poisoning Indicators Workgroup and General Injury ICD-10-CM Workgroup. CDC's guidance was informed by health departments and CSTE's workgroups and included several key aspects for assessing drug overdose in emergency department and hospitalisation discharge data. These include: (1) searching all diagnosis fields to identify drug overdose cases; (2) estimating drug overdose incidence using visits for initial encounter but excluding subsequent encounters and sequelae; (3) excluding underdosing and adverse effects from drug overdose incidence indicators; and (4) using codes T36-T50 for overdose surveillance. CDC's guidance also suggests analysing intent separately for ICD-10-CM coding.

Conclusions CDC's guidance provides health departments a key tool to better monitor drug overdoses in their community. The implementation and validation of this standardised guidance across all CDC-funded health departments will be key to ensuring consistent and accurate reporting across all entities.

\section{INTRODUCTION}

To combat the opioid overdose epidemic, the US Centers for Disease Control and Prevention (CDC) has focused on five key strategies: (1) conduct surveillance and research to monitor nonfatal and fatal overdose, (2) build state, local and tribal capacity to implement evidence-based approaches to prevent and respond to overdoses, (3) support providers, health systems and payers by providing them with the tools necessary to make evidencebased decision making related to opioid prescribing (eg, the CDC Guideline for Prescribing Opioids for Chronic Pain), ${ }^{1}$ (4) empower consumers to make safe choices on the use of prescription opioids ${ }^{2}$ and (5) improve communication and coordination between public health and public safety organisations. ${ }^{3}$ To improve standardisation and quality of nonfatal surveillance of opioid overdoses treated in emergency departments (EDs) and inpatient hospital settings (ie, CDC strategy 1), this paper presents CDC's guidance for using standard International Classification of Diseases, Tenth Revision, Clinical Modification (ICD-10-CM) definitions to identify and track ED visits and hospitalisations involving drug overdoses. These definitions were informed by work of the ICD-10-CM Drug Poisoning Indicators Workgroup, a partnership between CDC, the Council of State and Territorial Epidemiologists (CSTE) and health departments across the USA to enhance public health surveillance using ICD-10-CM hospital discharge codes.

Timely and high-quality data are crucial to help public health practitioners understand and respond to the opioid overdose epidemic confronting US communities. Historically, ED and hospitalisation discharge data have been the standard for tracking, monitoring and comparing the burden of non-fatal drug overdoses across states and localities. ${ }^{4}$ A key strength of ICD-10-CM discharge data is the universal use of the ICD-10-CM coding system to describe the following characteristics of ED visits and hospital inpatient admission: physician's confirmed diagnoses, medical procedures performed during the hospital visit and external causes of injury. Hospitals use ICD-10-CM codes to receive reimbursement from payers, including Medicare and other insurance providers for hospital visits and admissions. While the structure and data elements of ED datasets may vary across states and localities, most use the standard uniform billing (UB-04) formats developed by National Uniform Billing Committee and the American Hospital Association. ${ }^{5}$ The universal use of the standard codes in the ICD-10-CM allows standardised national, state and local surveillance of drug overdoses treated in hospitals that can be compared over time and across states and localities.

\section{Challenges to identifying ED visits and hospital inpatient admissions using ICD-10-CM}

Multiple challenges, however, impede the standard use of ICD-10-CM codes for drug overdose surveillance. A primary challenge is translating ED and inpatient drug overdose (referred to as 'poisoning' in the ICD-10-CM) definitions from International 


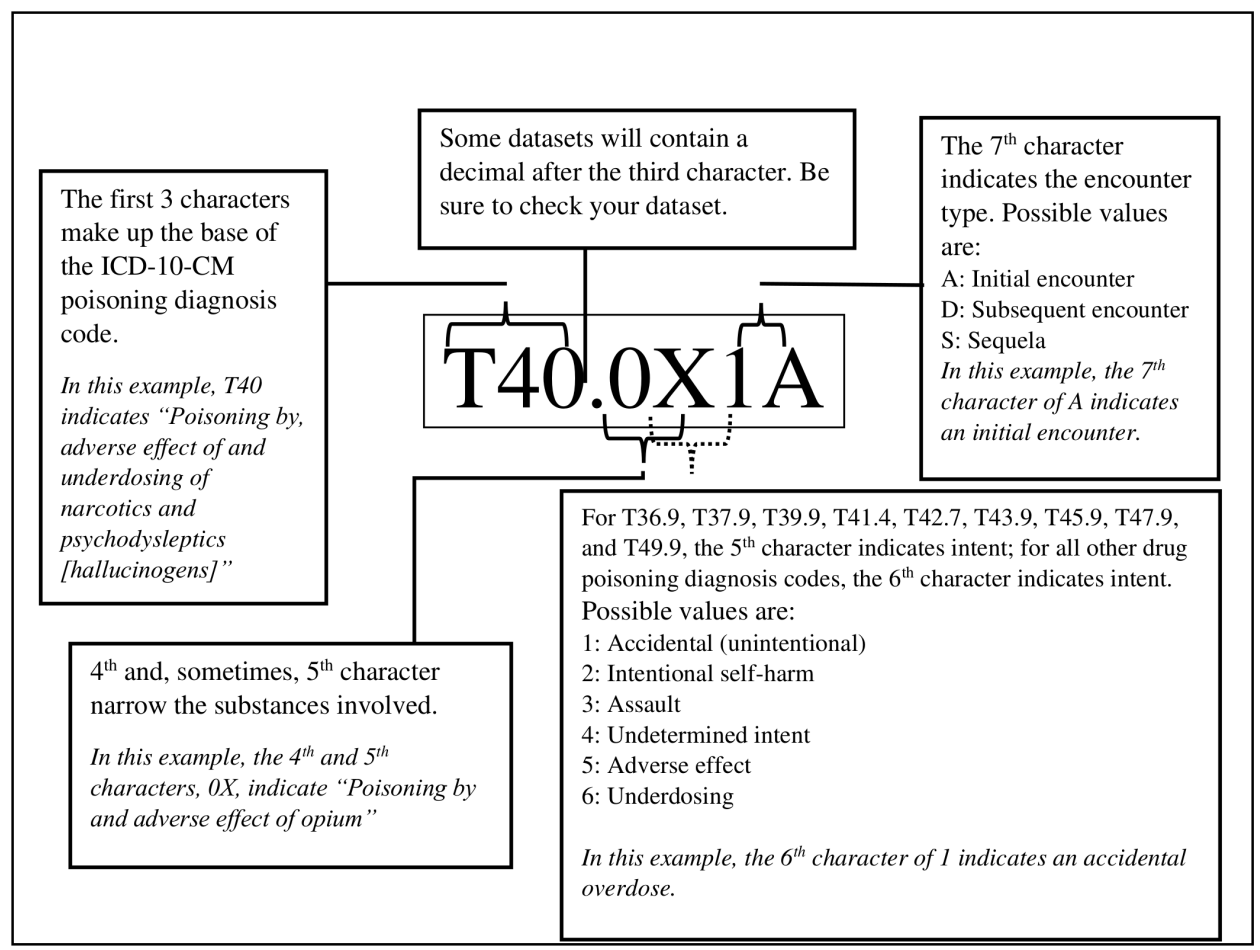

Figure 1 The anatomy of an ICD-10-CM drug poisoning diagnosis code. ICD-10-CM, International Classification of Diseases, 10th Revision, Clinical Modification.

Classification of Diseases, Ninth Revision, Clinical Modification (ICD-9-CM) to ICD-10-CM. On 1 October 2015, the US Department of Health and Human Services and Centers for Medicare \& Medicaid Services (CMS) mandated that all healthcare providers covered by the Health Insurance Portability and Accountability Act transition from ICD-9-CM coding to ICD-10-CM coding. ${ }^{6}$ The ICD-10-CM coding system is substantially different from the ICD-9-CM coding system (See figure 1 for a breakdown of an ICD-10-CM drug poisoning code). For instance, the number of injury diagnosis codes increased from 2600 to 43000 in the ICD-9-CM compared with the ICD-10-CM, while the number of external cause of injury codes increased from 1300 to $7500 .^{6}$ Thus, translation of ICD-9-CM drug overdose definitions to ICD-10-CM requires extensive conceptual work informed by empirical validation.

Several key changes in the diagnosis codes from ICD-9-CM to ICD-10-CM guidance had to be considered when defining and tracking drug overdose indicators. These include the following:

- External cause of injury codes for drug poisoning were combined with diagnostic codes in ICD-10-CM: under ICD-9-CM, poisoning surveillance indicators relied on both poisoning diagnosis codes, which described the substances involved, and poisoning external cause codes, which conveyed the intent (eg, suicide or unintentional). Under ICD-10-CM, the poisoning diagnosis codes or T-codes (T36T50) include information on both the substances involved and intent of the injury (ie, ' 1 ': accidental, ' 2 ': intentional self-harm, '3': assault, '4': undetermined, '5': adverse effect and ' 6 ': underdosing).

- Coding of undetermined and accidental poisonings changed in ICD-10-CM: under ICD-9-CM, medical coders were instructed to select undetermined intent for external causes of injury when the intent was unspecified in the medical record; however, the guidance under ICD-10-CM instructs coders to default to accidental intent and only select undetermined intent if the medical record explicitly indicates that the intent is undetermined. ${ }^{67}$

- ICD-10-CM introduced the concept of underdosing: ICD-10-CM introduced the concept of 'underdosing, ${ }^{8}$ the use of less than the prescribed drug according to a provider or drug manufacturer.

- ICD-10-CM introduced the concept of encounter type: ICD-10-CM introduced the new concept of encounter type for many injury diagnosis codes. For drug poisoning T-codes, a seventh character of 'A' indicates an initial encounter, ' $D$ ' indicates a subsequent encounter and ' $S$ ' indicates sequela. An initial encounter captures when a patient is receiving active treatment for the injury event, though it may not be the first encounter related to that injury event (eg, receiving treatment from two physicians for the same injury). A subsequent encounter captures when a patient is seen after active treatment for a follow-up related to the initial injury (eg, follow-up visit related to an overdose). A sequela captures when a patient is being treated for a secondary condition that arose because of the injury (eg, peripheral neuropathy).

Other challenges for identifying ED visits and hospitalisations involving drug overdose exist across both ICD-9-CM and ICD-10-CM coding. Data elements from these different data sources have several distinctions. Specifically, for hospital inpatient admissions, the first diagnosis field should identify the principal diagnosis, or 'the condition established after study to be chiefly responsible for occasioning the admission of the patient to the hospital for care'. However, for ED visits, a principal diagnosis code is not assigned and, instead visits are assigned a 'first-listed diagnosis' (ie, 'list first the ICD-10-CM code for the diagnosis, condition, problem, or other reason for encounter/visit shown in the medical record to be chiefly responsible for the services provided'). ${ }^{7}$ There is no national standard for ordering secondary diagnoses, and the number of secondary diagnosis fields varies widely among states and 
localities. Throughout this paper, the term 'primary diagnosis' will be used when referencing either the principal or first-listed diagnosis fields.

Standardisation of surveillance methods for drug overdose surveillance allows for comparisons across place and time and is crucial for programme monitoring, rigorous evaluation and outcomes research. ${ }^{10}$ Recognising the need to collect standardised surveillance data on drug overdoses, CDC collaborated with partners including the 42 states and the District of Columbia funded as part of the Prevention for States (PfS; https://www.cdc. gov/drugoverdose/states/state prevention.html), Data Driven Prevention Initiative (DDPI; https://www.cdc.gov/drugoverdose/ foa/ddpi.html) and Enhanced State Opioid Overdose Surveillance (ESOOS; https://www.cdc.gov/drugoverdose/foa/stateopioid-mm.html) programmes. This paper describes the process and final indicator definitions created for use with CDC overdose prevention and surveillance cooperative agreements. The implementation of these indicator definitions allows for standardised surveillance, evaluation and comparisons across states and localities funded by CDC.

\section{Developing standardised drug overdose surveillance definitions}

In 2016, to aid in the collection of standardised surveillance data on drug overdoses from PfS and DDPI partners, CDC proposed three provisional ICD-9-CM and ICD-10-CM based indicator definitions for non-fatal drug overdose to be used with both hospitalisation and ED discharge data sources: (1) all drug overdoses, (2) non-heroin opioid overdose and (3) heroin overdoses. ${ }^{11}$ Given the implementation of ICD-10-CM on 1 October 2015, the CDC did not have access to ICD-10-CM coded data with which to test and validate these drug overdose indicators. In 2017, in partnership to build injury surveillance methodologies in ICD-10-CM, CSTE and CDC launched the ICD-10-CM Drug Poisoning Indicators Workgroup to test the provisional indicator definitions and analyse drug overdose surveillance methodologies in ICD-10-CM. Concurrently, CDC funded the Kentucky Injury Prevention and Research Center to conduct a medical record review of the proposed PfS/DDPI drug overdose indicators using ED discharge data coded in ICD-10-CM ${ }^{12}$ and also funded 12 ESOOS states to analyse their state data to help further refine non-fatal overdose methodologies.

The ICD-10-CM Drug Poisoning Indicators Workgroup included members from CSTE, local and state health departments and CDC, who split into subgroups that completed an iterative process of indicator testing over approximately 2 years. As part of this process, 14 health departments analysed their states' hospital and ED discharge data coded in ICD-9-CM from 2010 to third quarter of 2015 and ICD-10-CM from fourth quarter of 2015 to 2017 . There were two major subgroups in the workgroup process. One group analysed trends across the transition from ICD-9-CM to ICD-10-CM, while the second group explored potential changes to the proposed indicator definitions. Four key questions drove the analyses for ED and hospitalisation discharge data:

1. How many diagnosis fields should be searched for drug poisoning indicators?

2. Which encounter types should be included or excluded?

3. Which manners of injury/intents should be included or excluded?

4. Should ICD-10-CM codes from outside of the injury chapter be included?
Current indicator guidance for drug overdose surveillance definitions

To inform the development of indicators for Drug Overdose Surveillance and Epidemiology (DOSE) ${ }^{13}$ CDC's non-fatal overdose surveillance system, we took into consideration the results from the ICD-10-CM Drug Poisoning Indicators Workgroup analyses as described in Tyndall Snow et al (in this issue). The final guidance, shown in table 1 , answers the four key questions described previously.

How many diagnosis fields should be searched for drug poisoning indicators? The analyses from eight states showed that among both hospitalisation and ED records, on average across states, over $20 \%$ of records containing drug overdose codes do not contain a drug overdose code in the primary diagnosis field. ${ }^{14}$ This suggests that potential cases could be missed by limiting the analysis to the primary diagnosis field. However, of concern was whether cases identified via a secondary diagnosis field reflect true overdose cases. Results from a CDC-funded medical record review of ED records that demonstrated the positive predictive value of ICD-10-CM opioid overdose diagnosis codes found in a secondary diagnosis field is relatively high at $76.5 \%$ for heroin overdose codes and $67.0 \%$ for codes representing opioid overdose other than heroin. ${ }^{12}$ Another advantage of searching all available fields is that it captures all drugs involved in a polydrug overdose, which allows individual drug poisoning indicators (eg, heroin overdose or stimulant overdose) to more accurately assess the magnitude of drug poisonings when more than one drug is involved. Also, an expanded definition has the benefit of parsimoniously including cases with a relevant primary diagnosis outside of the drug poisoning subset, for example, a case with a secondary diagnosis of drug overdose and a primary diagnosis of respiratory failure, a mental health condition or an $\mathrm{O}$-code for pregnancy complications from poisoning. CDC's guidance includes searching all available diagnosis fields.

Which encounter types should be included or excluded? Tyndall Snow and colleagues found that the frequency of subsequent encounters and sequelae among drug overdose codes found in any field were low among hospitalisation records across states and localities and lower among ED records. ${ }^{14}$ More important than the number of subsequent encounters and sequelae identified are the conceptual definitions of these encounter types and what they mean for drug poisoning incidence. According to the CMS-issued coding guidelines, subsequent encounters are 'encounters after the patient has completed active treatment of the condition and is receiving routine care for the condition during the healing or recovery phase'. ${ }^{15}$ Sequelae represent 'complications or conditions that arise as a direct result of a condition, such as scar formation after a burn'. ${ }^{15}$ In both cases, these visits do not represent new and acute overdose cases and will not help to approximate overdose incidence. CDC's guidance also excludes subsequent encounters and sequelae.

Which manners of injury/intent should be included or excluded? Underdosing codes make up a negligible proportion of drug T-codes, suggesting that the exclusion of these codes is unlikely to significantly affect rates. ${ }^{14}$ More importantly, underdosing, defined as 'taking less of a medication than is prescribed by a provider or a manufacturer's instruction', does not conceptually fit into drug overdose definitions. ${ }^{15}$ Adverse effect codes make up a considerable proportion of drug T-codes identified in Tyndall Snow and colleagues. ${ }^{14}$ The inclusion of adverse effects, which can represent clinical symptoms ranging from constipation to respiratory failure, has the potential to introduce a large number of false positives if included in the overdose 
Table 1 Guidance for ICD-9-CM and ICD-10-CM discharge diagnosis codes associated with emergency department visits and/or hospitalisations for all drug, all opioids, heroin and all stimulant overdose

\begin{tabular}{|c|c|c|c|c|}
\hline \multirow[b]{2}{*}{ Drug type } & \multirow[b]{2}{*}{$\begin{array}{l}\text { ICD-9-CM, any mention of diagnosis or external cause of } \\
\text { injury code }\end{array}$} & \multicolumn{3}{|l|}{ ICD-10-CM } \\
\hline & & Any mention of diagnosis & And a fifth/sixth character of: & $\begin{array}{l}\text { And a seventh character } \\
\text { of: }\end{array}$ \\
\hline All Drugs & $\begin{array}{l}\text { 960-979: poisoning by drugs, medicinal and biological } \\
\text { substances. } \\
\text { E850-E858: accidental poisoning by drugs, medicinal substances } \\
\text { and biologicals. } \\
\text { E950.0-E950.5: suicide and self-inflicted poisoning by solid or } \\
\text { liquid substances (note: this is analysed separately in OD2A* } \\
\text { guidance). } \\
\text { E980.0-E980.5: poisoning by solid or liquid substances } \\
\text { undetermined whether accidentally or purposely inflicted. } \\
\text { E962.0: assault by drugs and medicinal substances (note: } n o t \\
\text { included in OD2A* guidance). }\end{array}$ & $\begin{array}{l}\text { T36-T5: poisoning by drugs, medicaments and } \\
\text { biological substances. }\end{array}$ & $\begin{array}{l}\text { Intent is noted in the sixth character } \\
\text { of ICD-10-CM codes between T36 and } \\
\text { T50, with exceptionst where the fifth } \\
\text { character shows intent. } \\
\text { Character values: } \\
\text { 1: accidental (unintentional). } \\
\text { 2: intentional self-harm. } \\
\text { 3: assault. } \\
\text { 4: undetermined intent. } \\
\text { Does not include: } \\
\text { 5: adverse effect.§ }\end{array}$ & $\begin{array}{l}\text { A: initial encounter. } \\
\text { Does not include: } \\
\text { D: subsequent encounter. } \\
\text { S: sequela. }\end{array}$ \\
\hline All opioids & $\begin{array}{l}\text { 965.00: poisoning by opium. } \\
\text { 965.01: poisoning by heroin. } \\
\text { 965.02: poisoning by methadone. } \\
\text { 965.09: poisoning by other opiates and related narcotics. } \\
\text { E850.0: accidental poisoning by heroin. } \\
\text { E850.1: accidental poisoning by methadone. } \\
\text { E850.2: accidental poisoning by other opiates and related } \\
\text { narcotics. }\end{array}$ & $\begin{array}{l}\text { Before October } 12020 \text { : } \\
\text { T40.0X: poisoning by opium. } \\
\text { T40.1X: poisoning by heroin. } \\
\text { T40.2X: poisoning by other opioids. } \\
\text { T40.3X: poisoning by methadone. } \\
\text { T40.4X: poisoning by synthetic narcotics. } \uparrow \\
\text { T40.60: poisoning by unspecified narcotics. } \\
\text { T40.69: poisoning by other narcotics. } \\
\text { After } 1 \text { October } 2020 \text {, T40.4X will become: } \\
\text { T40.41: poisoning by fentanyl or fentanyl } \\
\text { analogues. } \\
\text { T40.42: poisoning by tramadol. } \\
\text { T40.49: poisoning by other synthetic narcotics. }\end{array}$ & 6: underdosing.§ & \\
\hline Heroin & $\begin{array}{l}\text { 965.01: poisoning by heroin. } \\
\text { E850.0: accidental poisoning by heroin. }\end{array}$ & T40.1X: poisoning by heroin. & & \\
\hline All stimulants & $\begin{array}{l}\text { 969.70: poisoning by psychostimulant, unspecified. } \\
\text { 969.71: poisoning by caffeine. } \\
\text { 969.72: poisoning by amphetamines. } \\
\text { 969.73: poisoning by methylphenidate. } \\
\text { 969.79: poisoning by other psychostimulants. } \\
\text { 970.0: poisoning by analeptics. } \\
\text { 970.81: poisoning by cocaine. } \\
\text { 970.89: poisoning by other central nervous system stimulants. } \\
\text { E854.2: accidental poisoning by psychostimulants. } \\
\text { E854.3: accidental poisoning by central nervous system } \\
\text { stimulants. } \\
\text { E855.2: accidental poisoning by local anaesthetics. }\end{array}$ & $\begin{array}{l}\text { T40.5X: poisoning by cocaine. } \\
\text { T43.60: poisoning by unspecified } \\
\text { psychostimulants. } \\
\text { T43.61: poisoning by caffeine. } \\
\text { T43.62: poisoning by amphetamines. } \\
\text { T43.63: poisoning by methylphenidate. } \\
\text { T43.64: poisoning by ecstasy. } \\
\text { T43.69: poisoning by other psychostimulants. }\end{array}$ & & \\
\hline
\end{tabular}

*OD2A is an acronym for CDC's Overdose Data to Action funding - https://www.cdc.gov/drugoverdose/od2a/index.html.

†The exception to this rule, where the fifth character denotes the intention: T36.9, T37.9, T39.9, T41.4, T42.7, T43.9, T45.9, T47.9 and T49.9.

¥Assault intents are not included in CDC's OD2A* guidance.

$\S$ When applicable, not all codes have an option for adverse effect or underdosing (eg, T40.1X does not include ' 5 ' or ' 6 ').

IOn 1 October 2020 revisions to this code will allow a more granular look at synthetic narcotic overdose - T40.4X will be replaced by three new codes: T40.41 (poisoning by fentanyl), T40.42 (poisoning by tramadol) and T40.49 (poisoning by other synthetic narcotics).

ICD-9-CM, International Classification of Diseases, Ninth Revision, Clinical Modification; ICD-10-CM, International Classification of Diseases, 10th Revision, Clinical Modification.

definition. The exclusion of adverse effects from overdose definitions is in keeping with historical epidemiological practice, as well as conceptual definitions laid out by CMS coding guidelines. ${ }^{15}{ }^{16}$ According to CMS coding guidelines, adverse effects codes should be used to describe 'an adverse effect of a drug that has been correctly prescribed and properly administered', while poisoning codes are used to describe 'a poisoning or reaction to the improper use of a medication (eg, overdose, wrong substance given or taken in error, wrong route of administration) ${ }^{\prime 15}$ The distinction between an adverse effect and an overdose does not rest on the clinical manifestation of symptoms but instead on how the drug was prescribed and administered. CDC's guidance excludes underdosing and adverse effect codes from overdose surveillance.

Should ICD-10-CM codes from outside of the injury chapter be included? Furthermore, Tyndall Snow and colleagues suggested that only codes T36-T50 be used for overdose surveillance with ICD-10-CM coded discharge data. These T-codes are part of a chapter in ICD-10-CM that represent injury and poisoning, more specifically 'poisoning by drugs, medicaments and biological substances'. The codes T51-T65, which represent 'toxic effects of substances chiefly nonmedicinal as to source' were not included in the definitions based on previous guidance from the Injury
Surveillance Workgroup (ISW7). ${ }^{17}$ In addition to ICD-10-CM codes T36-T50, several codes from $\mathrm{F}$ and $\mathrm{O}$ chapters came under consideration: F11-F19 with fifth and sixth characters of 12, 22 or 92 (these include mental and behavioural disorders due to substance use 'with intoxication' in the title), and O9A.2 (injury, poisoning and certain other consequences of external causes complicating pregnancy, childbirth and the puerperium). F-codes with the words 'with intoxication' were considered because of anecdotal reports that these codes were being mistakenly assigned to describe overdose events. While coder errors are possible, the inclusion of these codes in the indicator definitions without additional information from the medical record (eg, chief complaint text or triage notes) would likely introduce false positive cases. The O9A.2 code was considered because medical coders are required to sequence this code first in the medical record, followed by, in the event of an overdose, the appropriate poisoning code. ${ }^{15}$ Tyndall Snow and colleagues demonstrated that O9A.2 was very rare among overdose cases; however, searching all available diagnosis fields to identify appropriate codes from the $T$ chapter will capture these cases without explicitly searching for these O9A.2 as well. ${ }^{14} \mathrm{~A}$ complete list of CDC-recommended ICD-10-CM codes for drug overdose are included in the web materials, online supplemental appendix table A. 


\section{CONCLUSION}

With this guidance in place, CDC began implementation of the final indicator definitions in September 2019 with the initiation of the newly funded programme, OD2A. ${ }^{13}$ The OD2A programme includes 3-year funding for a total of 66 US states, territories and localities that focuses on the complex and changing nature of the opioid overdose epidemic and highlights the need for an interdisciplinary, comprehensive and cohesive public health approach. A subset of the 66 jurisdictions were funded to obtain high-quality, more comprehensive and timelier data on overdose morbidity and mortality and to use those data to inform prevention and response efforts (ie, 47 states and Washington DC). This timelier morbidity surveillance will be conducted through OD2A's DOSE system. The indicator surveillance definition provided in table 1 lays the foundation for all DOSE activities supported by OD2A. However, further guidance from OD2A's DOSE system requests that funded health departments report data on drug overdose incidence stratified by unintentional (accidental) and undetermined intent combined as compared with intentional self-harm (see online supplemental appendix table A). This differentiation will allow CDC and funded health departments the ability to better understand the intentionality attributed to drug overdoses and the burden associated with these different intents.

While standard and validated ICD-10-CM surveillance definitions increase the quality and comparability of indicators of drug overdose treated in EDs and hospital settings, drug overdose surveillance using ICD-10-CM data likely possesses many of the limitations previously documented with drug overdose surveillance conducted with ICD-9-CM. Limitations include low to moderate sensitivity to detect opioid overdoses with diagnoses codes in at least some hospitals ${ }^{18} 19$ and the imperfect sensitivity and specificity of rapid toxicology testing used in EDs and the inability of these toxicology tests to detect the full range of drugs involved in overdoses (eg, fentanyl or fentanyl analogues). ${ }^{20-22}$ These limitations require health departments to continually work to identify, account for, and improve the utility of these data. These also highlight the value of more in-depth toxicology testing of ED samples in response to outbreaks and complementary surveillance based on medical chart reviews. ${ }^{23}{ }^{24}$ Still, even with more in-depth toxicology testing, the lack of specificity of some diagnosis codes prohibit our ability to identify specific drugs. For example, prior to 1 October 2020, the ICD-10-CM T-code for 'poisoning by other synthetic narcotics' (T40.4X) included both illicit (eg, fentanyl) and prescribed (eg, tramadol, buprenorphine) opioids. However, on 1 October 2020 revisions to this code will allow a more granular look at synthetic narcotic overdose - T40.4X will be replaced by three new codes: T40.41 (poisoning by fentanyl), T40.42 (poisoning by tramadol) and T40.49 (poisoning by other synthetic narcotics). Also, the transition from ICD-9-CM to ICD-10-CM coding guidance also ${ }^{6}$ presents issues when assessing long-term trends in drug overdose. Lastly, health department access to these data sources and might vary; some may have the ability to analyse only one data source (eg, only hospitalisations and not ED visits), while others have access to both.

With assistance from our funded partners and CSTE collaborations, CDC will continue to test and evaluate these indicator surveillance definitions in addition to developing new drugrelated definitions integrating feedback from OD2A-funded health departments. In addition, as CMS releases coding modifications, including the introduction of new diagnosis codes, continued evaluation and validation are necessary to ensure CDC guidance stays relevant. The strong partnership between
CDC and its funded entities grows our knowledge of drug overdose-related $\mathrm{ED}$ visits and hospitalisations.

\section{What is already own on the subject}

- The transition to International Classification of Diseases, Tenth Revision, Clinical Modification (ICD-10-CM) coded data at the end of 2015 from the Ninth Revision (ICD-9-CM) added a substantial number of injury diagnosis codes.

- These changes required a translation of ICD-9-CM drug overdose definitions into the ICD-10-CM framework; however very little empirical validation has been conducted.

\section{What this study adds}

- This paper includes CDC's guidance for using standard ICD-10-CM code-based definitions to identify and track emergency department visits and hospitalizations involving drug overdoses.

- With these developed case definitions, CDC and health departments can test and evaluate the ICD-10-CM definitions for overdoses related to all drugs, opioids, heroin, and stimulants.

Acknowledgements Health departments participating in Centers for Disease Control and Prevention's (CDC) Prevention for States (PfS), Data Driven Prevention Initiative (DDPI) and Enhanced State Opioid Overdose Surveillance (ESOOS) programmes; the CDC PfS, DDPI and ESOOS team in the Division of Overdose Prevention, National Center for Injury Prevention and Control, CDC; and members of the Council of State and Territorial Epidemiologists International Classification of Diseases, 10th Revision, Clinical Modification (ICD-10-CM) Drug Poisoning Indicators Workgroup and General Injury ICD-10-CM Workgroup.

Collaborators The Overdose Morbidity Team author includes Felicita David, Brooke Hoots, Londell McGlone, Desiree M. Mustaquim, Cassandra M. Pickens, Douglas R. Roehler, Lawrence Scholl and Herschel Smith.

Contributors AV-K, SL and RMG conceived of the methodology, developed the case definition and drafted the manuscript; EP and PDM drafted several portions of the manuscript and the figure. The Overdose Morbidity Team (FD, BH, LM, DM, CP, $D R, L S$, and HS) helped develop the case definition and provided critical review of the manuscript. All authors critically reviewed and edited the manuscript and approved the final manuscript.

Funding The authors have not declared a specific grant for this research from any funding agency in the public, commercial or not-for-profit sectors.

Disclaimer The findings and conclusions in this paper of those of the authors and do not necessarily represent the official position of the Centers for Disease Control and Prevention.

\section{Competing interests None declared.}

Patient consent for publication Not required.

Provenance and peer review Commissioned; externally peer reviewed.

Data availability statement All data relevant to the study are included in the article or uploaded as supplementary information.

Supplemental material This content has been supplied by the author(s). It has not been vetted by BMJ Publishing Group Limited (BMJ) and may not have been peer-reviewed. Any opinions or recommendations discussed are solely those of the author(s) and are not endorsed by BMJ. BMJ disclaims all liability and responsibility arising from any reliance placed on the content. Where the content includes any translated material, BMJ does not warrant the accuracy and reliability of the translations (including but not limited to local regulations, clinical guidelines, terminology, drug names and drug dosages), and is not responsible for any error and/or omissions arising from translation and adaptation or otherwise.

Open access This is an open access article distributed in accordance with the Creative Commons Attribution Non Commercial (CC BY-NC 4.0) license, which permits others to distribute, remix, adapt, build upon this work non-commercially, and license their derivative works on different terms, provided the original work is 
properly cited, appropriate credit is given, any changes made indicated, and the use is non-commercial. See: http://creativecommons.org/licenses/by-nc/4.0/.

ORCID iD

Alana Vivolo-Kantor http://orcid.org/0000-0003-4576-1156

\section{REFERENCES}

1 Dowell D, Haegerich TM, Chou R. CDC Guideline for Prescribing Opioids for Chronic Pain - United States, 2016. MMWR Recomm Rep 2016;65:1-49.

2 Centers for Disease Control and Prevention. Rx awareness. Available: <https://www. cdc.gov/RxAwareness> [Accessed Sep 2019].

3 Centers for Disease Control and Prevention. CDC's Role in the Opioid Overdose Epidemic. Available: <https://www.cdc.gov/drugoverdose/prevention/cdc-role.html> [Accessed Sep 2019].

4 Council of State and Territorial Epidemiologists. Nonfatal opioid overdose standardized surveillance case definition. Available: <https://cdn.ymaws.com/www. cste.org/resource/resmgr/2019ps/final/19-CC-01_final_7.31.19.pdf> [Accessed Sep 2019].

5 Thomas KE, Johnson RL. State injury indicators report: Instructions for preparing 2013 data. Atlanta (GA): centers for disease control and prevention, National center for injury prevention and control, 2015.

6 Injury Surveillance Workgroup 9. The transition from ICD-9-CM to ICD-10-CM: guidance for analysis and reporting of injuries by mechanism and intent. Atlanta, GA: The Safe States Alliance, 2016

7 United States Department of Health and Human Services. ICD-10-CM official guidelines for coding and reporting, FY, 2017. Available: <https://www.cms.gov/ Medicare/Coding/ICD10/Downloads/2017-ICD-10-CM-Guidelines.pdf> [Accessed Sep 2019].

8 United States Department of Health and Human Services. ICD-10-CM official guidelines for coding and reporting, FY 2019. Available: <https://www.cdc.gov/nchs/ icd/data/10cmquidelines-FY2019-final.pdf> [Accessed Sep 2019].

9 Safe States. Collect and analyze injury and violence data. state of the states 2015 report. Available: <https://cdn.ymaws.com/www. safestates.org/resource/resmgr/ 2015sots/2015_SOTS_-_Issue_Brief_2___.pdf> [Accessed Oct 2019].

10 Office of Public Health Scientific Services. Cdc public health surveillance strategy report: 2014-2018. Atlanta, GA: Centers for Disease Control and Prevention, 2018.
11 CDC. CDC's Opioid Overdose Indicator Support Toolkit, Guidance for building and reporting on opioid-related mortality, morbidity, and PDMP Indicators, 2016.

12 Slavova S, Quesinberry D, Costich JF, et al. ICD-10-CM-Based definitions for emergency department opioid poisoning surveillance: electronic health record case confirmation study. Public Health Rep 2020;135:262-9.

13 Centers for Disease Control and Prevention. Overdose data to action. Available: $<$ https://www.cdc.gov/drugoverdose/od2a/index.html> [Accessed Sep 2019].

14 Tyndall Snow L, Hall KE, Custis C. Descriptive exploration of overdose codes in hospital and emergency department discharge data to inform development of drug overdose morbidity surveillance indicator definitions in ICD-10-CM. Inj Prev 2021:27:i27-34.

15 Centers for Medicare and Medicaid Services and the National Center for Health Statistics. ICD-10-CM official guidelines for coding and reporting FY 2019. Available: <https://www.cms.gov/Medicare/Coding/ICD10/Downloads/2019-ICD10-Coding Guidelines-.pdf> [Accessed Aug 2019].

16 Thomas KE, Johnson RL. State injury indicators report: Instructions for preparing 2015 data. Atlanta (GA): centers for disease control and prevention, National center for injury prevention and control, 2017.

17 Injury Surveillance Workgroup 7. Consensus recommendations for national and state poisoning surveillance. Atlanta, GA: The Safe States Alliance, 2012.

18 Reardon JM, Harmon KJ, Schult GC, et al. Use of diagnosis codes for detection of clinically significant opioid poisoning in the emergency department: a retrospective analysis of a surveillance case definition. BMC Emerg Med 2016;16:1-6.

19 Rowe C, Vittinghoff E, Santos G-M, et al. Performance measures of diagnostic codes for detecting opioid overdose in the emergency department. Acad Emerg Med 2017:24:475-83

20 Wu AHB. Limitations of point-of-care testing in the ED or ICU: a role for regional centralized toxicology laboratories. Clin Pharmacol Ther 2010;88:295-8.

21 Bhalla A. Bedside point of care toxicology screens in the ED: utility and pitfalls. Int J Crit IIIn Inj Sci 2014;4:257-60.

22 Dezman ZDW, Felemban W, Bontempo LJ, et al. Evidence of fentanyl use is common and frequently missed in a cross-sectional study of emergency department patients in Baltimore, Maryland. Clin Toxicol 2020;58:59-61.

23 Substance Abuse and Mental Health Services Administration. Drug abuse warning network. Available: <https://www.samhsa.gov/data/data-we-collect/dawn-drugabuse-warning-network> [Accessed Oct 2019]

24 Schillie SF, Shehab N, Thomas KE, et al. Medication overdoses leading to emergency department visits among children. Am J Prev Med 2009;37:181-7. 